\title{
Probing the gamma-ray source populations with photon count statistics and anisotropies
}

\author{
F. Donato ${ }^{1,2}$, N. Fornengo ${ }^{1,2}$, M. Korsmeier ${ }^{1,2,3}$, Silvia Manconi ${ }^{* 1,2}$, M. Regis ${ }^{1,2}$ \\ ${ }^{1}$ Dipartimento di Fisica, Università di Torino, via P. Giuria 1, 10125 Torino, Italy \\ ${ }^{2}$ Istituto Nazionale di Fisica Nucleare, Sezione di Torino, Via P. Giuria 1, 10125 Torino, Italy \\ ${ }^{3}$ Institute for Theoretical Particle Physics and Cosmology, RWTH Aachen University, \\ Sommerfeldstr. 16, 52056 Aachen, Germany \\ E-mail: manconieto.infn.it
}

\begin{abstract}
The global contribution of unresolved gamma-ray point sources to the extragalactic gamma-ray background has been recently measured using analyses employing the statistical properties of observed gamma-ray counts down to gamma-ray fluxes lower than those reached with standard source detection techniques. In addition, a new measurement of the angular power spectrum (APS) of the unresolved gamma-ray background was recently performed, using 8 years of data of the Fermi-LAT. However, the contribution from each specific source classes to the sourcecount distribution, such as blazars, misaligned Active Galactic Nuclei, or Star Forming Galaxies is affected by significant uncertainties, in particular in the unresolved flux regime. This translates into significantly different predictions for the source-count distribution as a function of energy. We here investigate the statistics of photon counts of more than 10 years of Fermi-LAT data and the new APS results to probe the extra-galactic gamma-ray source populations in different energy bands.
\end{abstract}

36th International Cosmic Ray Conference -ICRC2019-

July 24th - August 1st, 2019

Madison, WI, U.S.A.

\footnotetext{
* Speaker.
} 


\section{Introduction}

The Fermi-Large Area Telescope (LAT) [1] has enormously increased our knowledge of the nature of gamma-rays reaching the Earth. Gamma rays are produced in a wide ensemble of astrophysical sources, revealing non-thermal processes at play and cosmic particle accelerators. The all-sky gamma-ray emission observed by Fermi-LAT is typically divided in two main contributions: (i) a bright diffuse emission originated within our Galaxy, caused by the interaction of cosmic rays with interstellar gas, and interstellar radiation fields [2], and (ii) the Extragalactic Gamma-ray Background (EGB), which includes all the other sources of gamma rays outside the Galaxy [3]. The EGB is in turn decomposed in the emission coming from the individual sources of gamma rays, and the unresolved gamma-ray background (UGRB), what remains of the total measured gamma-ray emission after the subtraction of the resolved ${ }^{1}$ individual sources (see [4] for a review). This component is approximately isotropic on large angular scales, while anisotropies have been detected at small angular scales [5]. The UGRB anisotropies are typically attributed to the global contribution of one (or more) unresolved populations of point sources. Very recently, a new measurement of the angular power spectrum (APS) of the anisotropies in the UGRB has been performed, based on 8 years of Fermi-LAT data [6]. Apart from the contribution from unresolved faint point-sources, the UGRB might contain a contribution from diffuse components, such as cosmic-ray interactions with the intergalactic medium [7]. Most intriguingly, signals of annihilation or decay of dark matter (DM) particles in our Galactic halo, or in outer galaxies, can be hidden in the UGRB $[8,9]$. However, the searches for DM using the UGRB are hampered by significant uncertainties [10], connected to the modeling of the foreground emission from our Galaxy, and the uncertainties connected to the contribution from unresolved point sources.

Different source populations contribute to the EGB, as illustrated by the identified sources in the available catalogs $[11,12,13]$. Blazars are the most numerous population of individual extragalactic gamma-ray sources, see Refs. [14, 15, 16, 17, 18]. They constitute a specific class of Active Galactic Nuclei (AGN), strong electromagnetic emitters powered by the mass accretion onto central supermassive (larger than about $10^{6}$ solar masses) black holes in external galaxies [19]. The electromagnetic emission of AGNs is caused by several processes, and covers the range from radio to high-energy gamma-ray photons. Depending on the orientation of the relativistic jet of the active galaxy with respect to the observer, AGNs are divided in blazars and misaligned AGNs (mAGNs) [19]. The AGNs are classified as blazars when the jet is directed close to the line of sight [20]. Blazar are in turn sub-divided into two categories, depending on the radio luminosity and the morphology of the emission: BL Lacs have low radio luminosity, which is concentrated in the center and in the jets, while Flat Spectrum Radio Quasars (FSRQs) have high radio luminosities which is concentrated in the edge-elongated radio lobes [21]. They also have different photon indices, softer for the FSRQs ( 2.4) and harder ( 2.1) for the BL Lacs [22]. On the other hand, mAGNs are AGNs for which the viewing angle with respect to the jet is larger than 14 degrees [19]. Compared to blazars, they are expected to be less bright but more abundant [22]. In addition to AGN, the same mechanism causing the diffuse emission in the Milky Way, such as the interaction of cosmic rays in the interstellar gas and the interstellar radiation fields, is expected to produce

\footnotetext{
${ }^{1}$ We denote localized gamma-ray sources which have been observed (and included in the Fermi-LAT source cata$\operatorname{logs}$ ) as resolved sources. On the opposite, we refer to sources that are too faint to be detected individually as unresolved.
} 
gamma rays in other star-forming galaxies (SFGs). Being intrinsically faint but numerous, they are expected to significantly contribute to the observed gamma rays [23]. Only few galaxies of this type have been detected so far in gamma rays, e.g. the M31 and M33 [24].

The contribution to the EGB from these individual gamma-ray source populations can be characterized by their differential source count distribution $d N / d S$. This is the areal source density per solid angle element ${ }^{2}$, where $N$ is the number of sources in a given flux interval $(S, S+d S)$, and $S$ is the integral gamma-ray flux of a source in the considered energy bin. Typically, the $d N / d S$ for each source classes is characterized through the resolved point sources inserted in the catalogs. However, the amount of sources resolved in a given survey is limited by the detection threshold of the instrument. The $d N / d S$ of each source population can be, alternatively, obtained from data-driven models, derived from basic principles of source-intrinsic gamma-ray production, and by modeling the cosmological source evolution. This approach is based on the extrapolation of the $d N / d S$ inferred from different populations of resolved sources to the unresolved regime. The extrapolation of these models suggests that numerous sources with fluxes fainter than current source detection thresholds, such as blazars, mAGN, SFGs, and millisecond pulsars can possibly contribute to the observed EGB and UGRB [25, 18, 26, 22, 27, 23, 28]. Using this approach, in Ref. [9] it was demonstrated that the main source classes mentioned above can explain the UGRB spectrum, while other contributions, such as DM annihilation or decay, are constrained to be sub-dominant. However, the extrapolation of the gamma-ray $d N / d S$ to the unresolved flux regime is guided by correlations to the $d N / d S$ measurements at different wavelengths, and consequently suffers from significant uncertainties [4].

The dissection of the EGB composition is currently complemented by statistical methods. In fact, analyses employing the statistical properties of the observed gamma-ray counts map have recently measured the contribution from individual sources, and the diffuse EGB components, down to lower gamma-ray fluxes with respect to standard source-detection methods [29, 30, 31, 32, 33, 34]. In particular, in Refs. [32] and [35] (in the following Z16a and Z16b) it was shown that the 1-point Probability Distribution Function (1pPDF) of counts maps serves as a unique tool for precise measurements of the contribution from unresolved point sources to the gamma-ray sky, and the EGB's composition.

Within these statistical analysis, the contribution from unresolved point sources to the EGB is usually characterized by fitting the non-Poissonian contribution to photon counts with a generic multiple broken power law (MBPL) in photon $d N / d S$ (see Z16a and Z16b). As hinted in Z16b, the results of the generic $1 \mathrm{pPDF}$ analysis for the $d N / d S$ might already have the sensitivity to probe the extrapolation of the blazar models for the unresolved flux regime. The 1pPDF method in itself can be generalized in order to include a general parametrization for the fitted $d N / d S$. We perform, for the first time, a fit of Fermi-LAT data at $b>|30|$ deg with the 1pPDF method using a specific phenomenological models for describing the emission of the resolved and unresolved gamma-ray source populations, instead of a generic MBPL. Moreover, the angular correlations of the UGRB have been shown to constrain the models describing the emission from unresolved gamma-ray sources, in particular blazars, which are expected to dominate the anisotropy signal [36]. In this contribution, we outline how to constrain the gamma-ray source populations in the unresolved

\footnotetext{
${ }^{2} d \Omega$, omitted in our notation.
} 
regime, by combining complementary information coming from the analysis of $1 \mathrm{pPDF}$ and angular correlations. We discuss here our first preliminary results.

\section{Model for the blazar populations}

We consider the model of blazar population of Ref. [28], where a parametric description of the gamma-ray luminosity function, as a function of the redshift evolution and energy spectrum, has been constrained by using the properties of observed blazars. This model allows to combine FSRQs and BL Lacs into a single luminosity function. In particular, we consider the gamma-ray luminosity function $\Phi\left(L_{\gamma}, z, \Gamma\right)$, which is defined as the number of sources per unit of luminosity $L_{\gamma}$, of co-moving volume $V$, and of photon spectral index $\Gamma$. At redshift $z=0$ we define:

$$
\Phi\left(L_{\gamma}, z=0, \Gamma\right)=\frac{d N}{d L_{\gamma} d V d \Gamma}=\frac{A}{\ln (10) L_{\gamma}}\left[\left(\frac{L_{\gamma}}{L_{0}}\right)^{\gamma_{1}}+\left(\frac{L_{\gamma}}{L_{0}}\right)^{\gamma_{2}}\right]^{-1} \exp \left[-\frac{\left(\Gamma-\mu\left(L_{\gamma}\right)\right)^{2}}{2 \sigma^{2}}\right]
$$

where $A$ is an overall normalization, the indexes $\gamma_{1}$ and $\gamma_{2}$ rule the evolution of $\Phi$ with $L_{\gamma}$, and the last Gaussian term describes the distribution of the photon index $\Gamma$, with mean $\mu\left(L_{\gamma}\right)$ and width $\sigma$. The mean

$$
\mu\left(L_{\gamma}\right)=\mu^{*}+\beta\left[\log \left(\frac{L_{\gamma}}{\operatorname{erg~s}^{-1}}\right)-46\right]
$$

is defined by the constant term $\mu^{*}$, and weakly depends on luminosity through the $\beta$ parameter. We consider the redshift evolution of the gamma-ray luminosity function under the so-called Luminosity-Dependent Density Evolution (LDDE), $e\left(z, L_{\gamma}\right)$, which has been demonstrated to give the best description of the blazars observed by Fermi-LAT [28]. The luminosity function at all redshifts is finally given by $\Phi\left(L_{\gamma}, z, \Gamma\right)=\Phi\left(L_{\gamma}, z=0, \Gamma\right) \cdot e\left(z, L_{\gamma}\right)$ The observed energy spectrum of blazars $d N_{\gamma} / d E$ is described in terms of a broken power law:

$$
\frac{d N_{\gamma}}{d E_{\gamma}} \propto\left[\left(\frac{E_{\gamma}}{E_{b}}\right)^{\gamma_{a}}+\left(\frac{E_{\gamma}}{E_{b}}\right)^{\gamma_{b}}\right]^{-1} \exp \left[-\tau\left(E_{\gamma}, z\right)\right]
$$

where the $E_{b}$ correlates with $\Gamma$ as $\log \left(E_{b} / \mathrm{GeV}\right)=9.25-4.11 \Gamma$ [28]. The absorption of gamma-rays due to the interaction with the extragalactic background light [37] is modeled by the exponential factor in Eq. 2.3, which is treated here as in Ref. [38].

\subsection{Source-count distribution and APS from blazars}

Starting from the parametric description of the gamma-ray luminosity function of blazars outlined above, the differential source-count distribution $d N / d S$ is obtained as:

$$
\frac{d N}{d S}=\int_{0.01}^{5.0} d z \int_{1}^{3.5} d \Gamma \Phi\left[L_{\gamma}\left(S_{\mathrm{E}}, z, \Gamma\right), z, \Gamma\right] \frac{d V}{d z} \frac{d L_{\gamma}}{d S}
$$

where $L_{\gamma}\left(S_{\mathrm{E}}, z, \Gamma\right)$ is the luminosity of a source endowed with an energy flux $S_{E}$, localed at redshift $z$ and with spectral index $\Gamma$, being $S$ the flux in a specific energy bin. We note that the integration bounds for $\Gamma$ in Eq. 2.4 are such to properly cover the distribution of observed blazars, while the interval for the redshift $z$ is chosen to cover the interval in which we expect the vast majority of 
the emission [28]. Within the same parametric description of the blazar gamma-ray luminosity function, the angular power spectrum (ASP) for point-like blazars $C_{\mathrm{P}}^{i, j}$ is written as:

$$
\begin{aligned}
C_{\mathrm{P}}^{i, j}= & \int_{0.01}^{5.0} d z \frac{d V}{d z} \int_{1}^{3.5} d \Gamma \int_{L_{\min }}^{L_{\max }} d L_{\gamma} \Phi\left(L_{\gamma}, z, \Gamma\right) \times S_{i}\left(L_{\gamma}, z, \Gamma\right) S_{j}\left(L_{\gamma}, z, \Gamma\right) \\
& \times\left[1-\Omega\left(S_{\mathrm{E}}\left(L_{\gamma}, z, \Gamma\right), \Gamma\right)\right],
\end{aligned}
$$

where $i$ and $j$ label different energy bins. For $i=j$ we call the power spectrum auto-correlation APS, while we refer to the energy cross-correlation APS otherwise. The upper and lower bounds in the $L_{\gamma}$ integration are fixed to match the values in Ref. [28]. The term $\Omega(S, \Gamma)$ (often called "sky coverage") accounts for the Fermi-LAT sensitivity to resolve a given source, which depends on its photon flux $S$ and index $\Gamma$. This is estimated as explained in Ref. [36]. Moreover, we updated the $\Omega(S, \Gamma)$ computation outlined in Ref. [36], in order to account for the sensitivity of the latest Fermi-LAT source catalog catalog (4FGL) [11].

\section{Analysis setup}

The 1pPDF analysis follows the framework of Z16a,b, to which we refer for any further detail. The gamma-ray sky is modeled by adding three components: (i) an isotropic distribution of blazars point sources $d N / d S$, parameterized as Eq. 2.4; (ii) the Galactic diffuse emission, described by the spatial and spectral template provided by the Fermi-LAT collaboration (gll_iem_v06.fits), with a global free normalization $A_{\text {gal }}$; (iii) a truly isotropic component, which includes the sources too faint to be characterized with the $1 \mathrm{pPDF}$, or residual cosmic-ray contamination, with the integral flux $F_{\text {iso }}$ as a free normalization parameter.

We analyzed all-sky Fermi-LAT gamma-ray data from 2008 August 4 (239,557,417 s MET) through 2018 December 10 (566,097,546 s MET). We used Pass 8 data $^{3}$, along with the corresponding instrument response functions. The Fermi Science Tools (v10r0p5) ${ }^{4}$ were employed for event selection and data processing. The data selection referred to standard quality selection criteria (DATA_QUAL $==1$ and LAT_CONFIG==1), to values of the rocking angle of the satellite smaller than $52^{\circ}$, and maximum zenith angle of $90^{\circ}$. We selected events passing the ULTRACLEANVETO event class, and we use the corresponding instrument response functions. To avoid significant PSF smoothing, the event sample is restricted to the PSF 3 quartile (see Z16a,b). Data are analyzed in adjacent energy bands: (i) $1.04-1.99 \mathrm{GeV}$, (ii) $1.99-5.0 \mathrm{GeV}$, (iii) $5.0-10.4 \mathrm{GeV}$, (iv) 10.4-50.0 GeV, following Z16b, and binned using the HEALPix equal-area pixelization scheme [39] with $\kappa=7$. To avoid significant contamination from the diffuse emission of our Galaxy, we analyzed the data for $|b|>30 \mathrm{deg}$. The 1pPDF likelihood function is defined as the L2 method in Z16a. The nested sampling algorithm included in the MultiNest framework [40] is used to sample the posterior distribution, with 1500 live points together with a tolerance criterion of 0.2.

As for the APS fit, we exploit the recent measuremnt of Ref. [6]. We considered both the auto and cross-correlation APS for all twelve available energy bins from $0.5 \mathrm{GeV}$ to $1 \mathrm{TeV}$. Similar to the anlysis of the 1pPDF we use the nested sampling algorithm implemented in the MultiNest

\footnotetext{
${ }^{3}$ Publicly available at https://heasarc.gsfc.nasa.gov/FTP/fermi/data/lat/weekly/photon/

${ }^{4}$ https://fermi.gsfc.nasa.gov/ssc/data/analysis/software/
} 
package to perform a $\chi^{2}$-fit and to derive the best-fit parameter space of the model described in Sec. 2.

\section{Results}

To demonstrate that the 1pPDF analysis is properly working, we start by showing a simple fit of the blazar and FSRQ model, in which only the normalization is varied. We use the gamma-ray sky at $|b|>30 \mathrm{deg}$ and only photons of the first energy bin $1.04-1.99 \mathrm{GeV}$. The $d N / d S$ of the isotropic distribution of blazars point sources is parameterized as in Eq. 2.4. In Fig. 1 we show

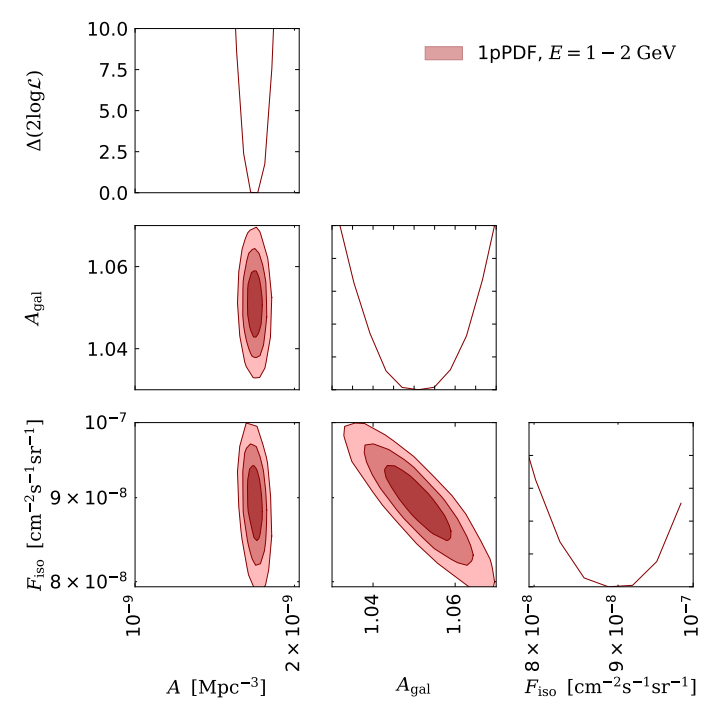

Figure 1: Triangle plot with the best-fit result of the 1pPDF method using the $d N / d S$ in the energy bin from $1-2 \mathrm{GeV}$. The contours show the $1-3 \sigma$ regions (frequentist interpretation) of the two-dimensional $\chi^{2}$ distribution for each combination of two parameters. The diagonal contains the likelihood profile for each of the parameters.

the marginalized posterior distributions of a fit where the free parameters are: the normalization of the blazar luminosity function $A$, the normalization of the Galactic diffuse emission $A_{\text {gal }}$ and the integral flux $F_{\text {iso }}$, see Sec. 3. When not varied, the parameters of the blazar luminosity function are fixed to the best fit obtained in Ref. [28], both for the 1pPDF and APS analysis. As visible from Fig. 1, the $1 \mathrm{pPDF}$ fits properly the $d N / d S$ parameterized as Eq. 2.4. In particular, the normalization of the blazar luminosity function in Eq. 2.4 converges towards $A=1.68 \pm 0.05 \times 10^{9} \mathrm{Mpc}^{-3}$. We are currently testing the $1 \mathrm{pPDF}$ fit in the different energy bins, also leaving more freedom to the blazar parametrization in Eq. 2.4. In Fig. 2 we report the result of the fit to the APS in terms of $C_{p}$. In the right panel, we show the Fermi-LAT measurement for the autocorrelation term of the $C_{p}$, together with the best fit result (solid line), and the $1 \sigma$ uncertainty (shaded band). We recall that the fit is performed in all the auto- and cross-correlation terms of the $C_{\mathrm{P}}^{i, j}$ in Eq. 2.5. The free parameters in this fit are the $A, \mu^{*}, \gamma_{1}$, and $z_{c}$. The first three parameters are defined in Eq. 2.1 and $z_{c}$ is the characteristic redshift of the LDDE (refer to [28] for details). The obtained model matches 

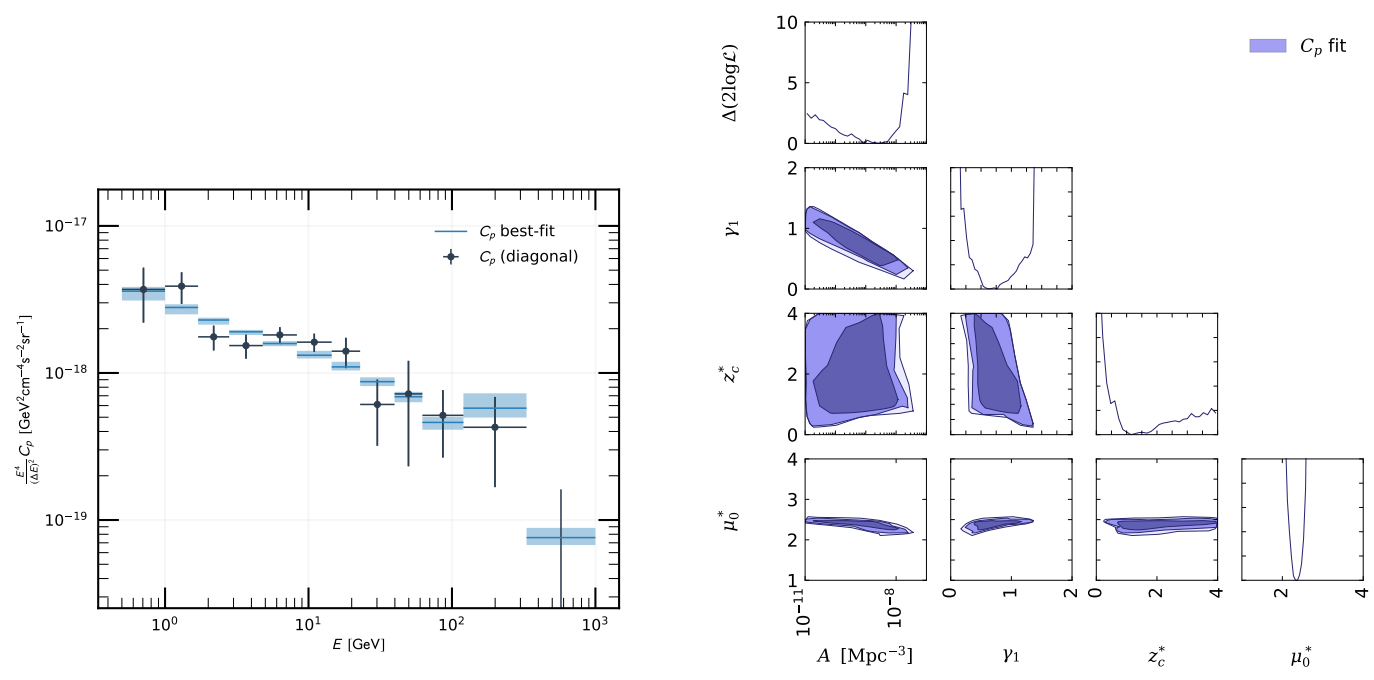

Figure 2: Left: Results for our fit to the angular correlations $C_{p}$, recently measured by Fermi-LAT [6] (black points), using the combined blazar and FSRQ model from [28]. For each energy bin, the best-fit result is reported with a blue solid line, while the shaded band indicates the $1 \sigma$ uncertainty. Right: Triangle plot of the $C_{p}$ fit. The contours show the $1-3 \sigma$ contours for each combination of two parameters. On the diagonal, we show the likelihood profile for each of the four fit parameters.

the $C_{p}$ measurement (auto- and cross correlation) very well. The triangle plot in the right panel of Fig. 2 shows the $1-3 \sigma$ best-fit contours of the fit of the APS.

\section{Conclusions}

We discussed a novel approach to constrain the contribution from the different gamma-ray source populations to the EGB, by combining the results coming from the 1pPDF analysis of more than 10 years of data with the recent analysis of the APS of the UGRB. Our preliminary results show that the two methods provide complementary constraints, which we will investigate with more details in the near future.

\section{References}

[1] W. B. Atwood, A. A. Abdo, M. Ackermann, et al., ApJ 697, pp. 1071-1102 (2009).

[2] M. Ackermann, M. Ajello, W. B. Atwood, et al., ApJ 750, pp. 3 (2012).

[3] M. Ackermann et al., Phys. Rev. Lett. 116(15), pp. 151105 (2016).

[4] Mattia Fornasa and Miguel A. Sánchez-Conde, Phys. Rept. 598, pp. 1-58 (2015).

[5] M. Ackermann, M. Ajello, A. Albert, et al., Phy. Rev. D 85(8), pp. 083007 (2012).

[6] M. Ackermann et al., Physical Review Letters 121(24), pp. 241101 (2018).

[7] D. Allard, Astroparticle Physics 39, pp. 33-43 (2012).

[8] Piero Ullio, Lars Bergstrom, Joakim Edsjo, et al., Phys. Rev. D66, pp. 123502 (2002). 
[9] Mattia Di Mauro and Fiorenza Donato, Phys. Rev. D91(12), pp. 123001 (2015).

[10] Torsten Bringmann, Francesca Calore, Mattia Di Mauro, et al., Phys. Rev. D89(2), pp. 023012 (2014).

[11] Fermi-LAT collaboration, arXiv:1902.10045 (2019).

[12] M. Ajello et al., Astrophys. J. Suppl. 232(2), pp. 18 (2017).

[13] Fermi-LAT collaboration, arXiv:1905.10771 (2019).

[14] Y. Inoue and T. Totani, ApJ 702, pp. 523-536 (2009).

[15] M. Ackermann, M. Ajello, A. Allafort, et al., ApJ 743, pp. 171 (2011).

[16] K. N. Abazajian, S. Blanchet, and J. P. Harding, Phy. Rev. D 84(10), pp. 103007 (2011).

[17] M. Ajello, M. S. Shaw, R. W. Romani, et al., ApJ 751, pp. 108 (2012).

[18] J. Singal, V. Petrosian, and M. Ajello, ApJ 753, pp. 45 (2012).

[19] C. M. Urry and P. Padovani, PASP 107, pp. 803 (1995).

[20] R. D. Blandford and M. J. Rees, Phys.S.Scr. 17, pp. 265-274 (1978).

[21] P. Padovani, D. M. Alexander, R. J. Assef, et al., A.A.Review 25, pp. 2 (2017).

[22] M. Di Mauro, F. Calore, F. Donato, et al., ApJ 780, pp. 161 (2014).

[23] I. Tamborra, S. Ando, and K. Murase, JCAP 9, pp. 043 (2014).

[24] M. Ackermann et al., ApJ 836, pp. 208 (2017).

[25] Y. Inoue, ApJ 733, pp. 66 (2011).

[26] M. Di Mauro, F. Donato, G. Lamanna, et al., Astrophys.J. 786, pp. 129 (2014).

[27] F. Calore, M. Di Mauro, and F. Donato, ApJ 796, pp. 14 (2014).

[28] M. Ajello, D. Gasparrini, M. Sánchez-Conde, et al., Astrophys.J. 800(2), pp. L27 (2015).

[29] S. Dodelson, A. V. Belikov, D. Hooper, et al., Phy. Rev. D 80(8), pp. 083504 (2009).

[30] D. Malyshev and D. W. Hogg, ApJ 738, pp. 181 (2011).

[31] M. R. Feyereisen, S. Ando, and S. K. Lee, JCAP 9, pp. 027 (2015).

[32] H.-S. Zechlin, A. Cuoco, F. Donato, et al., ApJS 225, pp. 18 (2016).

[33] M. Lisanti, S. Mishra-Sharma, L. Necib, et al., ApJ 832, pp. 117 (2016).

[34] Siddharth Mishra-Sharma, Nicholas L. Rodd, and Benjamin R. Safdi, Astron. J. 153(6), pp. 253 (2017).

[35] H.-S. Zechlin, A. Cuoco, F. Donato, et al., ApJL 826, pp. L31 (2016).

[36] Shin'ichiro Ando, Mattia Fornasa, Nicolao Fornengo, et al., Phys. Rev. D95(12), pp. 123006 (2017).

[37] I. V. Moskalenko, T. A. Porter, and A. W. Strong, ApJL 640, pp. L155-L158 (2006).

[38] A. Domínguez, J. R. Primack, D. J. Rosario, et al., MNRAS 410(4), pp. 2556-2578 (2011).

[39] K. M. Gorski, E. Hivon, A. J. Banday, et al., ApJ 622, pp. 759-771 (2005).

[40] F. Feroz, M. P. Hobson, and M. Bridges, MNRAS 398, pp. 1601-1614 (2009). 